\title{
OYUK YÜKLÜ KOMPAKT MİKROŞERİT ANTENLERİN REZONANS FREKANSININ HESAPLANMASINDA YSA VE BMSDUA YÖNTEMLERİNİN KULLANIMI
}

\author{
Ahmet KAYABAŞI ${ }^{1}$, Ali AKDAĞLI \\ ${ }^{1}$ Selçuk Üniversitesi, Silifke-Taşucu MYO, Elektronik Teknolojisi Programı, 33900, Taşucu, Mersin \\ ${ }^{2}$ Mersin Üniversitesi, Mühendislik Fakültesi, Elektrik-Elektronik Müh. Bölümü, Çiftlikköy, 33343, Mersin \\ ahmetkayabasi@selcuk.edu.tr, akdagli@mersin.edu.tr
}

(Geliş/Received: 12.12.2014; Kabul/Accepted: 22.10.2015)

\section{ÖZET}

\begin{abstract}
Mikroşerit antenler (MA), diğer mikrodalga antenlere olan bazı üstünlükleri nedeniyle kablosuz haberleşme sistemlerinde yaygın olarak kullanılmaktadırlar. MA'ların iletken kısımlarında modifikasyon yaparak kompakt mikroşerit antenler (KMA) elde edilir. KMA'lar aynı rezonans frekansı için geleneksel MA'lara göre daha küçük boyutlarda olmaları gibi bazı avantajlara sahiptirler. Literatürde, dikdörtgen halka şekilli KMA (DHKMA), C şekilli KMA (CKMA), E şekilli KMA (EKMA), H şekilli KMA (HKMA) ve L şekilli KMA'ların (LKMA) rezonans frekansı hesabı için yapay sinir ağları (YSA) ve/veya bulanık mantık sitemine dayalı uyarlanır ağ (BMSDUA) modelleri kullanılmıştır. Bu tarama çalışmasında, oyuk yüklü bu KMA’ların rezonans frekansı hesabı için kullanılan YSA ve BMSDUA modelleri ile elde edilen sonuçlar, literatürdeki diğer metotlar ile elde edilen sonuçlar ile karşılaştırılmış ve daha başarılı olduğu görülmüştür. Bu sonuçlara göre düzgün geometriye sahip olmayan oyuk yüklü KMA'ların rezonans frekansı hesabı için yapay zeka tekniklerinden YSA ve BMSDUA ağlarının başarılı bir şekilde kullanılabileceği görülmektedir. Ayrıca YSA ve BMSDUA çalışmaları incelendiğinde diğer metotlara göre daha hızlı ve etkili olduğu sonucu ortaya çıkmaktadır.
\end{abstract}

Anahtar Kelimeler: Mikroşerit anten, kompakt mikroşerit anten, H şekilli kompakt mikroşerit anten, rezonans frekansı, yapay sinir ağları, çok katmanlı algılayıcılar

\section{USAGE OF ANN AND ANFIS METHODS FOR COMPUTING RESONANT FREQUENCY OF SLOT-LOADED COMPACT MICROSTRIP ANTENNAS}

\begin{abstract}
Microstrip antennas (MAs) have been widely used in the wireless communication systems since they have some superiorities over other microwave antennas. Compact microstrip antennas (CMAs) are obtained by modifying the conductor parts of MAs. CMAs have some advantages over the conventional MAs such as smaller size for the same resonant frequency. The models of artificial neural network (ANN) and/or adaptive neuro-fuzzy inference system (ANFIS) have been used for computing the resonant frequency of rectangular ring shaped CMA (RRCMA), C shaped CMA (CCMA), E shaped CMA (ECMA), H shaped CMA (HCMA) and L shaped CMA (LCMA) in the literature. In this survey study, the results obtained by the ANN and ANFIS models used for the resonant frequency computation of these slot loaded CMAs were compared with those of the other methods in the literature and it is seen that these methods are more successful. According to these results, it is seen that ANN and ANFIS networks which are the artificial intelligence techniques can be successfully used for computing the resonant frequency of slot loaded CMA. Moreover, when the ANN and ANFIS studies were investigated, it appears that they are faster and more effective than the other methods.
\end{abstract}

Keywords: Microstrip antenna, compact microstrip antenna, resonant frequency, artificial neural network, adaptive neuro-fuzzy inference system

\section{GÍRISS (INTRODUCTION)}

Son yıllarda teknolojinin hızla gelişmesi ile birlikte kablosuz cihazların kullanımı her geçen gün artmakta ve boyutları küçülmektedir. Bu nedenle, söz konusu cihazlarda kullanılan antenlerden en önemlisi olan mikroşerit antenler (MA) yoğun ilgi görmektedir. Literatüre sunulan çalışmalar incelendiğinde, MA'lar 
için yapılan çalışmaların çoğu, analizlerinin kolaylığından dolayı dikdörtgen, üçgen ve daire gibi bilinen geometriler üzerinde yoğunlaşmıştır [1-7]. Bununla beraber, bilinen geometrilere sahip MA'ların boyutları, UHF (Ultra high frequncy) band uygulamaları için nispeten büyüktür. Bilinen MA'ların iletken kısımlarında modifikasyon yapmak suretiyle rezonans uzunluğunu artırmak ve bu sayede rezonans frekansını azaltmak için kompakt mikroşerit antenler (KMA) önerilmiştir [8-15]. Bu yöntem ile rezonans frekansı değişmeden KMA'nın iletken yama boyutları, MA'nın boyutlarına göre önemli oranda küçülmektedir. KMA'da anten boyutlarındaki küçülmeye karşılık, verim ve bant genişliği gibi bazı performans kriterlerinde düşüş olabilmektedir [3, 9]. Bilinen geometrik şekillere sahip MA'lar, transmisyon hattı modeli (transmission line model) [16] ve boşluk modeli (cavity model) [17] gibi yöntemlerle analitik olarak analiz edilebilmektedir. Bununla beraber, geometrilerinin düzgün olmamasından dolayı bu yöntemlerle KMA'ların analitik olarak analizi oldukça zordur. $\mathrm{Bu}$ nedenle, KMA'ların tasarımında ve analizinde, genellikle elektromanyetik nümerik hesaplama yöntemleri kullanılır. Başlıca elektromanyetik nümerik hesaplama yöntemleri, moment metodu (Method of Moment - MoM) [18] ve zaman domeninde Maxwell denklemlerinin çözümünü sağlayan zamanda sonlu farklar domeni (Finite Difference Time Domain FDTD) [19] metodudur. Literatürde değişik yapılara sahip KMA'ların rezonans frekanslarının belirlenmesinde, birbirinden farklı doğruluk ve basitlikte yöntemler sunulmuştur [3, 8, 11-15]. Önerilen yöntemlerde, dikdörtgen MA'lar (DMA) için yaygın olarak kullanılan etkin dielektrik sabiti ve iletken boyundaki uzamayı hesaplayan ifadeler kullanılmıştır. $\mathrm{Bu}$ yöntemlerin kullanılması formüllerin sayısını artırmakta ve hesaplama süresini nispeten uzatmaktadır. KMA'ların elektriksel (dielektrik sabiti) ve fiziksel (anten boyutları) parametreleri kullanılarak, antenin rezonans frekansının belirlenmesi, yüksek derecede doğrusal olmayan bir problemdir. $\mathrm{Bu}$ nedenle, rezonans frekansının nümerik yöntemlerle hesaplanması için anten parametreleri kullanılarak çok sayıda benzetim yapılır. MA'ların elektronik iletişim pazarında kullanımının artması, performans analizinin yapılmasında daha basit metotların kullanılmasını gerektirmektedir. Bundan dolayı MA tasarımciları, çok fazla hesaplama zamanı gerektirmeyen basit yaklaşımları tercih etmektedirler. Bilgisayar teknolojilerinin son yıllardaki hızlı gelişimi ile birlikte yapay sinir ağları (YSA) ve bulanık mantık sistemine dayalı uyarlanır ağ (BMSDUA) gibi yapay zekaya dayanan teknikler, klasik optimizasyon ve geleneksel analitik yaklaşımlardan daha esnek ve elverişli sonuçlar üreten güçlü alternatif araçlar olmuştur [4-6]. YSA ve BMSDUA, MA'ların rezonans frekansının hesaplanmasında çokça kullanılmıştır [5-6, 20-22, 24, 27]. Bu tarama çalışmasında; farklı geometrik şekillerdeki KMA'ların rezonans frekansının hesaplanmasinda YSA ve BMSDUA modellerini kullanarak sonucu kısa sürede hesaplayan basit yaklaşımlar incelenmiştir.

\section{MIKKROŞERIT ANTENLER (MICROSTRIP ANTENNAS)}

Diğer mikrodalga antenlere olan üstünlükleri nedeniyle araştırmacıların ilgi odağı haline gelen MA'lar, bugüne kadar çok büyük gelişme kaydetmiştir. Küçük hacimleri, üretimlerinin kolay olması, maliyetinin düşük olması ve çift bant özelliklerine sahip olmaları gibi avantajlarından dolayı güdümlü füzeler, roketler, uydular gibi sadece askeri uygulamalarda kullanılan MA'lar, günümüzde kablosuz haberleşmenin hemen hemen her alanında kullanılmaktadırlar [1-3]. Literatürde MA için yapılan çalışmalar, Şekil 1'de görülen dikdörtgen, üçgen ve daire gibi bilinen geometriler üzerinde yoğunlaşmıştır [1-3, 4-7].

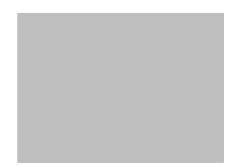

(a)

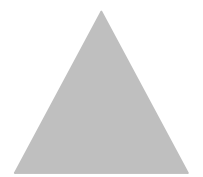

(b)

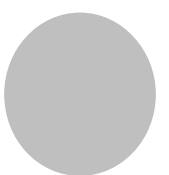

(c)
Şekil 1. Geleneksel MA geometrileri (The geometries of traditional MA)

\section{OYUK YÜKLÜ KOMPAKT MIKROŞERITT ANTENLER (SLOT LOADED COMPACT MICROSTRIP ANTENNAS)}

MA'larda, iletken yama veya toprak düzleminde boşluk açma (slot loading) ve kısa devre pin gibi çeşitli modifikasyon yöntemleri kullanılarak daha düşük rezonans frekansında çalışan antenler elde etmek mümkündür. DMA'nın kenar(lar)ında oluşturulan girintilerin etkisiyle (slot loading) antenin etkin uzunluğu artmakta, böylece rezonans frekansı azalmaktadır. $\mathrm{Bu}$ şekilde küçültülen antenlere KMA denilmektedir. $\mathrm{Bu}$ bölümde, YSA ve BMSDUA modellerinin oyuk yüklü KMA'ların rezonans frekansı hesabı için literatüre sunulan çalışmalar taranmış ve literatüre sunulan diğer metotlara göre başarıları değerlendirilmiştir. Bu kapsamda, E şekilli KMA (EKMA) ve $\mathrm{H}$ şekilli KMA (HKMA) için YSA, dikdörtgen halka şekilli KMA (DHKMA) ve C şekilli KMA (CKMA) için BMSDUA ve L şekilli KMA (LKMA) için ise hem YSA hem de BMSDUA modellerinin literatüre sunulduğu görülmektedir. $\mathrm{Bu}$ çalışmalarda, YSA ve BMSDUA ağlarının eğitimi ve testi için, farklı fiziksel ve elektriksel parametrelere sahip belirli sayıda KMA'nın benzetimleri elektromanyetik benzetim paket programları kullanılarak yapılmıştır. Bu kapsamda, CKMA [37] ve HKMA [38] benzetimleri FDTD metodunu kullanan XFDTD elektromanyetik benzetim programı ile, EKMA [39], LKMA [40] DHKMA [41] benzetimleri ise MoM kullanan IE3D ${ }^{\mathrm{TM}}$ elektromanyetik benzetim programı ile gerçekleştirilmiş ve antenlerin rezonans frekansları elde edilmiştir. İncelenen çalışmalarda, KMA'ların rezonans frekansı hesabı için Şekil 2'de görülen ileri 
beslemeli, geri yayılımlı, ÇKA'ya dayanan YSA modeli kullanılmıştır. ÇKA'nın eğitiminde, bir çok mühendislik uygulamasında kullanılan ve hızlı bir optimizasyon algoritması olan Levenberg-Marguart (LM) tercih edilmiştir. KMA'ların rezonans frekansı hesabı için ise Şekil 3'de görülen Sugeno tip bulanık çıkarım sistemi (BÇS) temelli BMSDUA modeli kullanılmış ve BMSDUA ağına ait parametreler hibrit öğrenme algoritması ile güncellenmiştir. YSA ve BMSDUA ağlarına, benzetimleri yapılan KMA'ların boyutları ve dielektrik sabiti giriş olarak, rezonans frekansı ise çıkış olarak verilmiştir. Ağların, eğitim ve test hataları Eşitlik 1'de verilen ortalama yüzde hata $(\mathrm{OYH})$ ifadesi ile hesaplanmıştır. YSA ve BMSDUA ağlarının başarılarını, benzetim verilerinin dışında test etmek için belirli sayı ve şekillerde gerçekleştirilen KMA'ların ölçüm sonuçları ile karşılaştırılmış ve başarılı sonuçlar alındığı görülmüştür [37-41].

$O Y H=\frac{\sum\left|\frac{f_{X F D T D / I E 3 D}-f_{Y S A / B M S D U A}}{f_{X F D T D / I E 3 D}}\right| x 100}{\text { Toplam anten saylst }}$

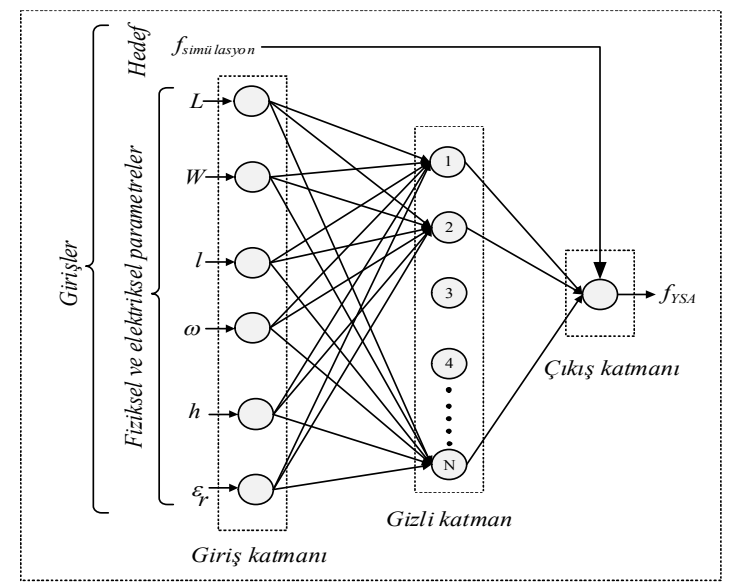

Şekil 2. KMA'ların rezonans frekansı hesabı için kullanılan YSA modeli (ANN model used for computing the resonant frequency of CMAs)

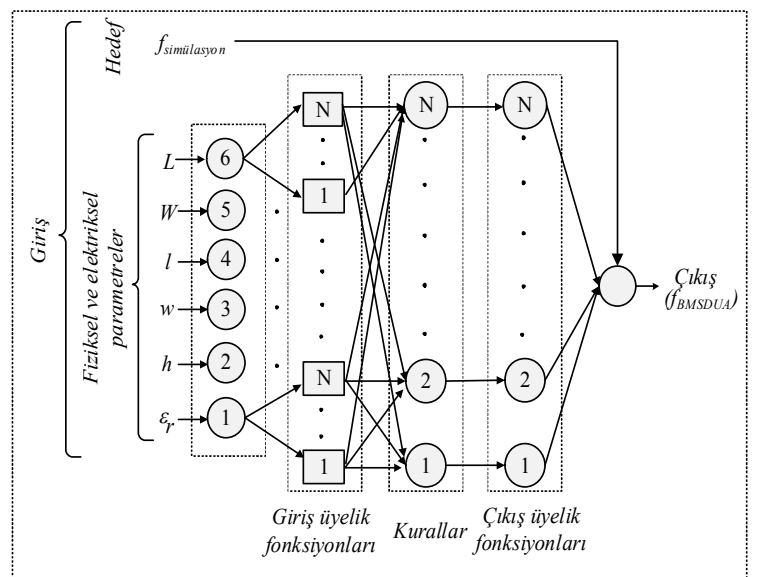

Şekil 3. KMA'ların rezonans frekansı hesabı için kullanılan BMSDUA modeli (ANFIS model used for computing the resonant frequency of CMAs)

\subsection{C Şekilli Kompakt Mikroşerit Anten (C Shaped Compact Microstrip Antennas)}

Bir CKMA, Şekil 4'de gösterildiği gibi, bir DMA'dan, onun 1şıma yapmayan kenarı boyunca simetrik olarak $(l x w)$ boyutlarında açılan bir boşlukla elde edilir.
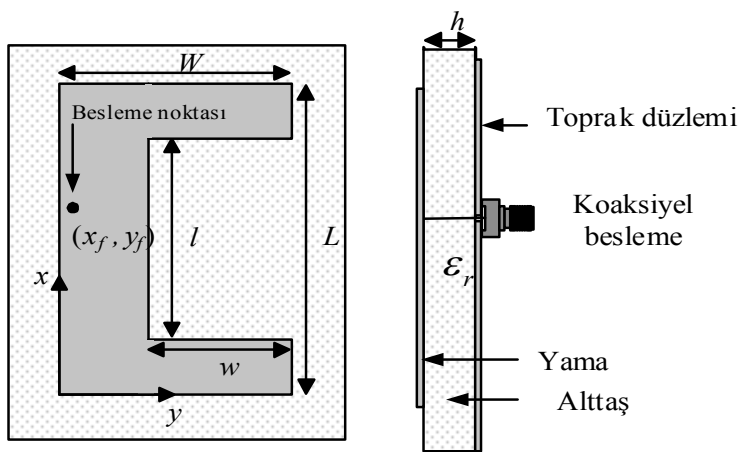

Şekil 4. CKMA geometrisi (Geometry of CCMA)

Literatürde CKMA'ların rezonans frekansı hesabı için belirli sayıda benzetim verileri kullanılarak, etkin dielektrik sabiti ve iletken boyundaki uzamayı hesaplayan formüller [11, 14-15] ve yapay zeka tekniklerinden BMSDUA'nın kullanıldığı [37] yaklaşımlar önerilmiştir. Literatürde CKMA'ların rezonans frekansı hesabı için Eşitlik 2'de [15] yerine konularak kullanılan, iletken boyundaki uzamayı hesaplayan formüllerden bir tanesi Eşitlik 3'de [15] örnek olarak verilmiştir.

$$
\begin{aligned}
& f_{r_{X}}=\frac{c}{2 L_{X e f f} \sqrt{\varepsilon_{r}}} \\
& L_{\text {Ceff }}=0.351 L+0.5\left(\frac{L+s}{\varepsilon_{r}^{0.033}}\right)\left(\frac{W}{h}\right)^{0.08} \\
& +2.922 d\left(\frac{d}{L}\right)-0.436 s\left(\frac{s}{W}\right)
\end{aligned}
$$

Akdagli vd. [37] tarafindan 2011 yılında yapılan çalışmada rezonans frekansı hesabı için Tablo 1'de parametreleri verilen BMSDUA modeli kullanılmıştır.

Tablo 1. CKMA'nın rezonans frekansı hesabında kullanılan BMSDUA parametreleri (The ANFIS parameters used for computing the resonant frequency of CCMAs)

\begin{tabular}{lc}
\hline Parametreler & Tip/değer \\
\hline Giriș üyelik fonksiyonu & Gaussian \\
Çıkș üyelik fonksiyonu & Linear \\
Giriş sayısı & 6 \\
Çıkşs sayı1sı & 1 \\
Bulannk kurallar sayısı & 16 \\
Üyelik fonksiyonlar sayısı & 16 \\
Epok & 50 \\
Etki aralığı & 0,5 \\
S1kıştırma faktörü & 1,25 \\
Kabul oranı & 0,5 \\
Geri çevirme oranı & 0,15 \\
Nonlineer parametre sayısı & $6 \times 16 \times 2=192$ \\
Lineer parametre sayısı & $7 \times 16=112$ \\
Düğ̈üm sayısı & 233 \\
Eğitim veri çifti sayısı & 129 \\
\hline
\end{tabular}


Benzetimi yapılan 129 CKMA, BMSDUA ağını eğitmek için kullanılmış ve eğitim için OYH değeri \% 0,841 olarak elde edilmiştir. BMSDUA modelini test etmek için kullanılan 15 CKMA verisi için rezonans frekansı sonuçları Tablo 2'de verilmiştir. Tablo 2'den OYH değerinin \%1,259 olarak hesaplandığ görülmektedir. BMSDUA kullanılarak yapılan bu çalışma, benzetim sonuçları ve Deshmukh ve Kumar, 2007 [11], Akdağlı vd., 2011 [14] ve Toktas vd., 2011 [15] tarafindan önerilen formüller ile elde edilen sonuçlar ile de karşılaştırılmış ve sonuçları Tablolar 3 ve Tablo 4'de verilmiştir. Tablo 3 ve Tablo 4'de, BMSDUA ile elde edilen sonuçların literatürde önerilen formülasyon [11, 16, 15] sonuçlarına göre daha başarılı olduğu görülmektedir. Ayrıca, bu çalışmada önerilen BMSDUA modeli, aynı çalışmada laboratuvar ortamında gerçeklenmiş CKMA sonucu ile de test edilmiştir. Tablo 5'de, gerçeklenen CKMA için benzetim, ölçüm ve BMSDUA ile elde edilen rezonans frekans değerleri görülmektedir.

Tablo 2. Test süreci için BMSDUA ile belirlenen CKMA rezonans frekans değerleri (The resonant frequencies of CCMAs determined by ANFIS for test process)

\begin{tabular}{|c|c|c|c|c|c|c|c|c|c|}
\hline \multirow{2}{*}{$\begin{array}{l}\text { Anten } \\
\text { sayis1 }\end{array}$} & \multicolumn{5}{|c|}{ Yama boyutları (mm) } & \multirow[b]{2}{*}{$\varepsilon_{r}$} & \multicolumn{2}{|c|}{ Rezonans frekansları $(\mathrm{GHz})$} & \multirow{2}{*}{$\begin{array}{l}\text { Yüzdelik } \\
\text { hata }(\%)\end{array}$} \\
\hline & $L$ & $W$ & $l$ & $w$ & $h$ & & Benzetim [37] & BMSDUA [37] & \\
\hline 1 & 30 & 20 & 15 & 7 & 1,6 & 2,33 & 2,654 & 2,643 & 0,399 \\
\hline 2 & 30 & 20 & 7 & 15 & 1,6 & 4,28 & 1,380 & 1,346 & 2,435 \\
\hline 3 & 30 & 20 & 20 & 7 & 1,6 & 4,28 & 2,017 & 2,020 & 0,139 \\
\hline 4 & 30 & 20 & 12 & 15 & 1,6 & 9,80 & 0,902 & 0,886 & 1,807 \\
\hline 5 & 30 & 20 & 20 & 15 & 1,6 & 9,80 & 0,956 & 0,915 & 4,289 \\
\hline 6 & 60 & 40 & 30 & 20 & 3 & 2,33 & 1,164 & 1,152 & 1,014 \\
\hline 7 & 60 & 40 & 20 & 9 & 3 & 4,28 & 1,081 & 1,086 & 0,444 \\
\hline 8 & 60 & 40 & 40 & 20 & 3 & 4,28 & 0,887 & 0,891 & 0,440 \\
\hline 9 & 60 & 40 & 30 & 9 & 3 & 9,80 & 0,721 & 0,721 & 0,055 \\
\hline 10 & 60 & 40 & 40 & 30 & 3 & 9,80 & 0,471 & 0,468 & 0,658 \\
\hline 11 & 90 & 60 & 20 & 13 & 6 & 2,33 & 0,970 & 0,997 & 2,794 \\
\hline 12 & 90 & 60 & 40 & 30 & 6 & 2,33 & 0,776 & 0,777 & 0,116 \\
\hline 13 & 90 & 60 & 20 & 40 & 6 & 4,28 & 0,527 & 0,525 & 0,304 \\
\hline 14 & 90 & 60 & 60 & 40 & 6 & 4,28 & 0,527 & 0,524 & 0,664 \\
\hline 15 & 90 & 60 & 60 & 13 & 6 & 9,80 & 0,499 & 0,516 & 3,327 \\
\hline OYH & & & & & & & & & 1,259 \\
\hline
\end{tabular}

Tablo 3. Benzetim verileri [11] için BMSDUA ile elde edilen CKMA rezonans frekansları (The resonant frequencies of CCMA obtained by ANFIS for simulation data [11])

\begin{tabular}{|c|c|c|c|c|c|c|c|c|}
\hline \multirow{2}{*}{\multicolumn{2}{|c|}{$\begin{array}{c}\text { Boşluk boyutları } \\
(\mathrm{mm})\end{array}$}} & \multicolumn{7}{|c|}{ Rezonans frekansları $(\mathrm{GHz})$} \\
\hline & & \multirow{3}{*}{$\begin{array}{c}\text { Benzetim } \\
{[11]}\end{array}$} & \multirow{3}{*}{$\begin{array}{c}\text { BMSDUA } \\
{[37]} \\
\end{array}$} & \multicolumn{5}{|c|}{ Hesaplanan } \\
\hline \multirow{2}{*}{$l$} & \multirow{2}{*}{$w$} & & & \multirow{2}{*}[15]{} & \multirow{2}{*}[14]{} & \multicolumn{3}{|c|}{ [11] } \\
\hline & & & & & & $\# 1$ & $\# 2$ & $\# 3$ \\
\hline 5 & 5 & 1,562 & 1,603 & 1,562 & 1,657 & 1,502 & 1,630 & - \\
\hline 10 & 10 & 1,445 & 1,429 & 1,445 & 1,497 & 1,398 & 1,408 & - \\
\hline 15 & 15 & 1,286 & 1,275 & 1,286 & 1,334 & 1,309 & 1,241 & - \\
\hline 20 & 20 & 1,130 & 1,125 & 1,130 & 1,178 & 1,231 & 1,111 & 1,002 \\
\hline 25 & 25 & 0,991 & 0,991 & 0,991 & 1,035 & 1,164 & 1,008 & 0,928 \\
\hline 40 & 30 & 0,899 & 0,901 & 0,899 & 0,924 & - & 0,893 & 0,856 \\
\hline 5 & 30 & 0,929 & 0,931 & 0,929 & 0,963 & - & 1,029 & 0,904 \\
\hline 10 & 30 & 0,887 & 0,901 & 0,887 & 0,938 & - & - & 0,896 \\
\hline 2 & 30 & 0,964 & 0,953 & 0,964 & 0,982 & - & - & 0,910 \\
\hline
\end{tabular}

Tablo 4. Benzetim verileri [11] için hesaplanan yüzdelik hatalar (The percentage errors calculated for simulation data [11])

\begin{tabular}{|c|c|c|c|c|c|c|c|}
\hline \multicolumn{2}{|c|}{ Boşluk boyutları (mm) } & \multirow{3}{*}{ BMSDUA [37] } & \multicolumn{5}{|c|}{ Yüzdelik hatalar (\%) } \\
\hline \multirow{2}{*}{$l$} & \multirow{2}{*}{$w$} & & \multirow{2}{*}[15]{} & \multirow{2}{*}[14]{} & \multicolumn{3}{|c|}{ [11] } \\
\hline & & & & & $\# 1$ & $\# 2$ & $\# 3$ \\
\hline 5 & 5 & 2,612 & 0 & 6,082 & 3,841 & 4,353 & - \\
\hline 10 & 10 & 1,107 & 1,315 & 3,599 & 3,253 & 2,561 & - \\
\hline 15 & 15 & 0,855 & 0,467 & 3,732 & 1,788 & 3,499 & - \\
\hline 20 & 20 & 0,451 & 0,531 & 4,248 & 8,938 & 1,681 & 11,33 \\
\hline 25 & 25 & 0,010 & 0,908 & 4,440 & 17,46 & 1,715 & 6,357 \\
\hline 40 & 30 & 0,245 & 1,001 & 2,781 & - & 0,667 & 4,783 \\
\hline 5 & 30 & 0,248 & 0,215 & 3,660 & - & 10,764 & 2,691 \\
\hline 10 & 30 & 1,567 & 2,706 & 5,750 & - & - & 1,015 \\
\hline 2 & 30 & 1,151 & 1,867 & 1,867 & - & - & 5,602 \\
\hline $\mathrm{OYH}$ & & 0,916 & 1,001 & 1,151 & 7,055 & 3,605 & 5,296 \\
\hline
\end{tabular}


Tablo 5. Gerçeklenen CKMA'nın [37] benzetim, ölçüm ve BMSDUA sonuçları (The results of simulation, measurement and ANFIS for fabricated CCMA [37])

\begin{tabular}{|c|c|c|c|c|c|c|c|c|}
\hline \multicolumn{5}{|c|}{ Yama boyutları (mm) } & \multirow[b]{2}{*}{$\varepsilon_{r}$} & \multicolumn{3}{|c|}{ Rezonans frekansı (GHz) } \\
\hline $\mathrm{L}$ & $\mathrm{W}$ & $l$ & $w$ & $h$ & & Benzetim [37] & Ölçüm [37] & BMSDUA [37] \\
\hline 30 & 20 & 5 & 20 & 1,57 & 2,33 & 2,870 & 2,930 & 2,900 \\
\hline
\end{tabular}

Tablo 6. HKMA'nın rezonans frekansı hesabında kullanılan YSA parametreleri (The ANN parameters used for computing the resonant frequency of HCMAs)

\begin{tabular}{lc}
\hline Parametreler & Değer \\
\hline Giriş sayısı & 6 \\
Çıkış sayısı & 1 \\
Epok sayısı & 250 \\
Minimum gradient azalması & $10^{-10}$ \\
Momentum katsayısı $(\mu)$ & 0,0001 \\
$\mu$ değerindeki artış & 4 \\
$\mu$ değerindeki düşüş & 0,1 \\
Maksimum $\mu$ değeri & $10^{10}$ \\
\hline
\end{tabular}

3.2 H Şekilli Kompakt Mikroşerit Antenler (H Shaped Compact Microstrip Antennas)

Bir HKMA Şekil 5'de görüldüğü gibi DMA'nın iki kısa kenarı boyunca $(l x w)$ boyutlarında açılmış iki simetrik ve eşit boşlukla gerçekleştirilmiştir [3, 11]. HKMA için literatürde iletken boyundaki uzamayı hesaplayan formüller kullanılarak rezonans frekansı hesabı yapan yaklaşımlar mevcuttur $[11,15]$. İletken boyundaki uzamayı hesaplayan formüllerden biri Eşitlik 2'deki [15] rezonans frekansı formülünde yerine konulmak üzere Eşitlik 4'te [15] verilmiştir.

$$
\begin{aligned}
& L_{\text {Heff }}=(0.912 L-0.643 s)\left(\frac{s}{d}\right)^{0.437} \\
& +W\left(\frac{L}{d}\right)^{0.726}+\frac{h}{\varepsilon_{r}}-0.445 L\left(\frac{W}{d}\right)
\end{aligned}
$$

Kayabasi vd. [38] 2011 yılında, HKMA'nın rezonans frekansı hesabı için Tablo 6'da ağ parametreleri verilen, 3 nöronlu 1 gizli katmandan oluşan ve daha başarılı sonuçlar veren bir YSA modeli önermişlerdir. Benzetimi yapılan 216 HKMA'nın 196'sın1, YSA ağını eğitmek için kullanmışlar ve eğitim için $\mathrm{OYH}$ değerini \% 0,68 olarak hesaplamışlardır.

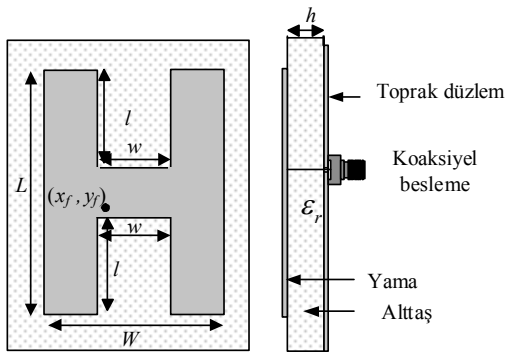

Şekil 5. HKMA geometrisi (Geometry of HCMA)

Tablo 7. Test sürecinde YSA ile belirlenen HKMA rezonans frekansları (The resonant frequencies of HCMA

\begin{tabular}{|c|c|c|c|c|c|c|c|c|c|}
\hline \multirow{2}{*}{$\begin{array}{l}\text { Anten } \\
\text { say1s1 }\end{array}$} & \multicolumn{5}{|c|}{ Anten boyutları (mm) } & \multirow[b]{2}{*}{$\varepsilon_{r}$} & \multicolumn{2}{|c|}{ Rezonans frekansı (GHz) } & \multirow{2}{*}{$\begin{array}{c}\text { Yüzdelik hata (\%) } \\
\mathrm{H}_{\mathrm{YSA}}[38]\end{array}$} \\
\hline & $L$ & $W$ & $l$ & $w$ & $h$ & & Benzetim [38] & YSA [38] & \\
\hline 1 & 30 & 30 & 6 & 14 & 1,59 & 2,5 & 1,645 & 1,648 & 0,182 \\
\hline 2 & 30 & 30 & 14 & 6 & 1,06 & 2,5 & 2,389 & 2,373 & 0,669 \\
\hline 3 & 30 & 30 & 22 & 6 & 0,53 & 2,5 & 2,866 & 2,869 & 0,104 \\
\hline 4 & 30 & 30 & 22 & 14 & 1,59 & 10,2 & 1,380 & 1,359 & 1,521 \\
\hline 5 & 30 & 30 & 22 & 22 & 1,59 & 4,5 & 2,123 & 2,122 & 0,047 \\
\hline 6 & 30 & 40 & 10 & 6 & 2,12 & 2,5 & 1,751 & 1,750 & 0,057 \\
\hline 7 & 30 & 40 & 20 & 6 & 1,59 & 2,5 & 2,282 & 2,284 & 0,087 \\
\hline 8 & 30 & 40 & 30 & 22 & 1,59 & 2,5 & 2,760 & 2,776 & 0,579 \\
\hline 9 & 30 & 40 & 20 & 14 & 0,53 & 2,5 & 2,176 & 2,187 & 0,505 \\
\hline 10 & 30 & 40 & 10 & 14 & 1,59 & 4,5 & 1,274 & 1,241 & 2,590 \\
\hline 11 & 40 & 40 & 10 & 30 & 2,12 & 2,5 & 1,433 & 1,438 & 0,348 \\
\hline 12 & 40 & 40 & 20 & 10 & 1,59 & 2,5 & 1,805 & 1,795 & 0,554 \\
\hline 13 & 40 & 40 & 30 & 20 & 1,06 & 2,5 & 2,070 & 2,069 & 0,048 \\
\hline 14 & 40 & 40 & 10 & 20 & 0,53 & 2,5 & 1,327 & 1,329 & 0,150 \\
\hline 15 & 40 & 40 & 10 & 30 & 1,59 & 10,2 & 0,743 & 0,728 & 2,018 \\
\hline 16 & 50 & 50 & 12,5 & 12,5 & 2,12 & 2,5 & 1,114 & 1,118 & 0,359 \\
\hline 17 & 50 & 50 & 12,5 & 25 & 1,59 & 2,5 & 1,061 & 1,073 & 1,131 \\
\hline 18 & 50 & 50 & 12,5 & 37,5 & 1,06 & 2,5 & 1,167 & 1,154 & 1,114 \\
\hline 19 & 50 & 50 & 37,5 & 12,5 & 0,53 & 2,5 & 1,698 & 1,699 & 0,058 \\
\hline 20 & 50 & 50 & 37,5 & 37,5 & 1,59 & 10,2 & 0,849 & 0,889 & 4,711 \\
\hline YH & & & & & & & & & 0,840 \\
\hline
\end{tabular}
determined by ANN for test process) 
YSA modelini test etmek için kullanılan 20 HKMA verisi için rezonans frekansı sonuçları Tablo 7'de görülmektedir. Tablo 7'de görüldüğü gibi $\mathrm{OYH}$ değeri $\% \quad 0,840$ olarak hesaplanmıştır. YSA kullanılarak yapılan çalışma, literatürdeki benzetim sonuçları [9] ve deneysel [10] veriler kullanılarak da test edilmiştir. YSA ile elde edilen sonuçlar, ayrica, Akdağlı ve Toktaş, 2010 [13] tarafindan HKMA'lar için önerilen formül sonuçları ile de karşılaştırılmış ve karşılaştırma sonuçları Tablo 8'de verilmiştir. Ayrıca bu çalışmada sunulan yaklaşım, aynı çalışma içerisinde deneysel olarak ölçülmüş HKMA verileri ile de test edilmiş ve sonuçlar Tablo 9'da görüldüğü gibi başarılı bir şekilde elde edilmiştir.

\subsection{E Sekilli Kompakt Mikroserit Antenler (E} Shaped Compact Microstrip Antennas)

EKMA boyutları $(L \times W)$ olan DMA'nın uzun kenarların birinde, $(l \times w)$ boyutlarında eşit ve merkeze göre simetrik iki boşluğun açılmasıyla elde edilen $\mathrm{E}$ şekilli yamanın, toprak düzlem üzerinde bulunan $h$ yüksekliğindeki $\varepsilon_{r}$ dielektrik sabitine sahip alttaş malzemenin üzerine yerleştirilmesiyle Şekil 6'da gösterildiği gibi elde edilmiştir [42, 43]. Literatürde EKMA için yapılan çalışmalarda besleme noktası seçimine bağlı olarak, DMA'ya göre daha yüksek rezonans frekansı ve geniş bant elde eden ve yaklaşık rezonans frekansı formülleri öneren çalışmalar mevcuttur [43-46]. Ayrıca DMA'ya göre daha düșük rezonans frekansına sahip, daha küçük boyut hedeflenerek yapılmış ve etkin uzunluk ifadesi formülünün önerildiği çalışmalarda vardır [47]. Eşitlik 2'de yerine konulmak üzere etkin uzunluk ifadesinin kullanıldığı formül Eşitlik 5'te [47] verilmiştir.
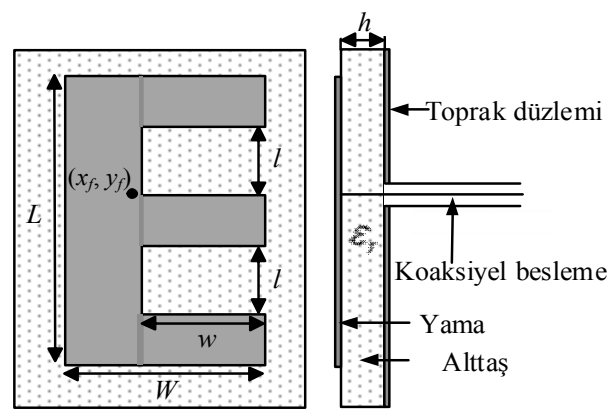

Şekil 6. EKMA geometrisi (Geometry of ECMA)

$$
\begin{aligned}
& L_{\text {Eeff }}=\left(1.321 L-0.677 l_{s}\right)\left(\frac{l_{s}}{L}\right)^{0.086} \\
& +1.693 W\left(\frac{w_{s}}{W}\right)^{1.974}+\frac{6.371 h}{\varepsilon_{r}^{2.458}}
\end{aligned}
$$

Akdağlı vd. [39] tarafindan 2013 yılında yapılan çalışmada, DMA'ya göre daha küçük boyut amaçlanarak yapılmış ve EKMA'ların rezonans frekansı hesabı için Tablo 10'da ağ parametreleri verilen ve 3 nöronlu 1 gizli katmandan oluşan YSA modelini kullanan bir yaklaşım önerilmiştir.

Tablo 8. Benzetim [9] ve ölçüm [10] verileri için YSA ile elde edilen HKMA rezonans frekansları ve sonuçların karşılaştırılması (The resonant frequencies of HCMA determined by ANN for simulation [9] and measurement [10]

\begin{tabular}{|c|c|c|c|c|c|c|c|c|c|c|}
\hline \multirow{2}{*}{$\begin{array}{l}\text { Anten } \\
\text { say1s1 }\end{array}$} & \multicolumn{4}{|c|}{ Anten boyutları (mm) } & \multirow{2}{*}{$\varepsilon_{r}$} & \multicolumn{3}{|c|}{ Rezonans frekansları $(\mathrm{GHz})$} & \multicolumn{2}{|c|}{ Yüzdelik hata (\%) } \\
\hline & $L$ & $W$ & $l$ & $w$ & & {$\left[9^{*}\right],\left[10^{\Delta}\right]$} & YSA [38] & [13] & YSA [38] & [13] \\
\hline 1 & 54,6 & 33 & 33 & 26 & 2,5 & $1,740^{*}$ & 1,741 & 1,774 & 0,058 & 1,954 \\
\hline 2 & 54,6 & 33 & 10 & 26 & 2,5 & $1,140^{*}$ & 1,132 & 1,107 & 0,702 & 2,895 \\
\hline 3 & 32,69 & 33 & 15 & 4,09 & 2,5 & $2,170^{*}$ & 2,216 & 2,270 & 2,120 & 4,608 \\
\hline 4 & 43,59 & 33 & 15 & 14,99 & 2,5 & $1,590^{*}$ & 1,586 & 1,613 & 0,252 & 1,447 \\
\hline 5 & 49,04 & 33 & 15 & 20,44 & 2,5 & $1,410^{*}$ & 1,423 & 1,436 & 0,922 & 1,844 \\
\hline 6 & 54,6 & 33 & 15 & 26 & 2,5 & $1,290^{*}$ & 1,309 & 1,298 & 1,473 & 0,620 \\
\hline 7 & 35,54 & 33 & 15 & 26 & 2,5 & $1,970^{*}$ & 2,013 & 2,022 & 2,183 & 2,640 \\
\hline 8 & 45,08 & 33 & 15 & 26 & 2,5 & $1,550^{*}$ & 1,590 & 1,574 & 2,581 & 1,548 \\
\hline 9 & 64,16 & 33 & 15 & 26 & 2,5 & $1,130^{*}$ & 1,115 & 1,109 & 1,327 & 1,858 \\
\hline 10 & 75 & 33 & 15 & 26 & 2,5 & $0,960^{*}$ & 0,971 & 0,955 & 1,146 & 0,521 \\
\hline 11 & 54,6 & 23 & 15 & 26 & 2,5 & $1,510^{*}$ & 1,479 & 1,498 & 2,053 & 0,795 \\
\hline 12 & 54,6 & 28 & 15 & 26 & 2,5 & $1,390^{*}$ & 1,391 & 1,390 & 0,072 & 0,000 \\
\hline 13 & 24 & 38 & 11 & 8 & 2,2 & $2,190^{\Delta}$ & 2,200 & 2,199 & 0,411 & 1,781 \\
\hline $\mathrm{OYH}$ & & & & & & & & & 1,180 & 1,730 \\
\hline
\end{tabular}
data and comparison of the results)

Tablo 9. Gerçeklenen HKMA'nın benzetim, ölçüm ve YSA sonuçları (The results of simulation, measurement and

\begin{tabular}{|c|c|c|c|c|c|c|c|c|}
\hline \multicolumn{5}{|c|}{ Yama boyutları (mm) } & \multirow[b]{2}{*}{$\varepsilon_{r}$} & \multicolumn{3}{|c|}{ Rezonans frekansı $(\mathrm{GHz})$} \\
\hline$L$ & $W$ & $l$ & $w$ & $h$ & & Benzetim [38] & Ölçüm [38] & YSA [38] \\
\hline 29 & 30,02 & 15 & 6,5 & 1,57 & 2,33 & 2,500 & 2,505 & 2,535 \\
\hline
\end{tabular}
ANN for fabricated HCMA) 
Tablo 10. EKMA'nın rezonans frekansı hesabında kullanılan YSA parametreleri (The ANN parameters used for computing the resonant frequency of ECMAs)

\begin{tabular}{lc}
\hline Parametreler & Değer \\
\hline Girişsayısı & 6 \\
Çıkss sayısı & 1 \\
Epok sayısı & 250 \\
Seed değeri & 7559532 \\
Minimum gradient azalması & $10^{-10}$ \\
Momentum katsayısı $(\mu)$ & 0,0001 \\
$\mu$ değerindeki artış & 4 \\
$\mu$ değerindeki düşüş & 0,1 \\
Maksimum $\mu$ değeri & $10^{10}$ \\
\hline
\end{tabular}

Tablo 11. YSA [39] ve formül [47] ile hesaplanan rezonans frekans değerleri ve yüzdelik hatalar (The resonant frequencies and percentage errors determined by ANN [39] and formula [47])

\begin{tabular}{|c|c|c|c|c|c|c|c|c|c|c|}
\hline \multicolumn{6}{|c|}{ Yama boyutları $(\mathrm{mm})$} & \multicolumn{3}{|c|}{ Rezonans frekansları (GHz) } & \multicolumn{2}{|c|}{ Yüzdelik hata (\%) } \\
\hline$L$ & $W$ & $l$ & $w$ & $h$ & $\varepsilon_{r}$ & $\begin{array}{c}\text { Benzetim } \\
\text { [39] }\end{array}$ & $\begin{array}{c}\text { Formül } \\
{[47]}\end{array}$ & $\begin{array}{l}\text { YSA } \\
{[39]}\end{array}$ & $\begin{array}{c}\text { Formül } \\
{[47]}\end{array}$ & $\begin{array}{l}\text { YSA } \\
\text { [39] }\end{array}$ \\
\hline 25 & 20 & 6 & 4 & 1,57 & 2,33 & 3,490 & 3,474 & 3,492 & 0,455 & 0,066 \\
\hline 25 & 20 & 4 & 8 & 1,57 & 2,33 & 2,970 & 3,005 & 2,972 & 1,188 & 0,071 \\
\hline 25 & 20 & 8 & 4 & 1,57 & 2,33 & 3,524 & 3,548 & 3,542 & 0,668 & 0,516 \\
\hline 25 & 20 & 6 & 4 & 1,57 & 4,5 & 2,587 & 2,592 & 2,578 & 0,185 & 0,332 \\
\hline 25 & 20 & 2 & 12 & 1,57 & 6,15 & 1,597 & 1,594 & 1,603 & 0,215 & 0,382 \\
\hline 32,5 & 25 & 7,5 & 5 & 2,5 & 2,33 & 2,660 & 2,646 & 2,675 & 0,513 & 0,560 \\
\hline 32,5 & 25 & 2,5 & 10 & 2,5 & 4,5 & 1,739 & 1,750 & 1,728 & 0,628 & 0,615 \\
\hline 32,5 & 25 & 7,5 & 20 & 2,5 & 4,5 & 1,170 & 1,159 & 1,157 & 0,952 & 1,103 \\
\hline 32,5 & 25 & 7,5 & 20 & 2,5 & 6,15 & 1,009 & 0,995 & 1,003 & 1,413 & 0,644 \\
\hline 40 & 30 & 3 & 20 & 3,17 & 2,33 & 1,500 & 1,489 & 1,492 & 0,744 & 0,533 \\
\hline 40 & 30 & 3 & 26 & 3,17 & 4,5 & 0,899 & 0,890 & 0,886 & 1,013 & 1,446 \\
\hline 25 & 20 & 8 & 8 & 1,57 & 4,5 & 2,310 & 2,293 & 2,301 & 0,719 & 0,403 \\
\hline 40 & 30 & 3 & 6 & 3,17 & 6,15 & 1,400 & 1,406 & 1,405 & 0,437 & 0,371 \\
\hline 40 & 30 & 9 & 12 & 3,17 & 6,15 & 1,220 & 1,218 & 1,223 & 0,203 & 0,279 \\
\hline OYH & & & & & & & & & 0,667 & 0,523 \\
\hline
\end{tabular}

Tablo 12. Gerçeklenen EKMA'nın benzetim, ölçüm ve YSA sonuçları (The results of simulation, measurement and ANN for fabricated ECMA)

\begin{tabular}{|c|c|c|c|c|c|c|c|c|}
\hline \multicolumn{4}{|c|}{ Yama boyutları (mm) } & \multicolumn{2}{|c|}{ Besleme noktası } & \multicolumn{3}{|c|}{ Rezonans frekansları $(\mathrm{GHz})$} \\
\hline $\mathrm{L}$ & W & $l$ & $w$ & $x_{f}$ & $y_{f}$ & Benzetim [39] & Ölçüm [39] & YSA [39] \\
\hline 25,00 & 20,00 & 7,47 & 13,03 & 6,45 & 14,23 & 2,400 & 2,407 & 2,396 \\
\hline
\end{tabular}

Bu çalışmada, benzetimleri yapılan 144 EKMA'dan 130’u YSA modelinin eğitiminde kullanılmış ve eğitim neticesinde $\mathrm{OYH}$ değeri \% 0,257 olarak hesaplanmıştır. Geriye kalan ve eğitim sürecinde ağın görmediği 14 anten ise YSA modelinin testinde kullanılmıştır. Test neticesinde elde edilen sonuçlar Tablo 11'de, Toktaş ve Akdağlı, 2012 [47] tarafindan önerilen formül sonuçları ile de karşılaştırılmıştır. Bu çalışma ile literatüre sunulan YSA modeli [39], aynı çalışma için gerçeklenmiş olan EKMA verileri ile de test edilmiş ve Tablo 12'de görüldüğü gibi başarılı bir sonuç elde edilmiştir.

\subsection{L Şekilli Kompakt Mikroșerit Antenler (L Shaped Compact Microstrip Antennas)}

LKMA, Şekil 7'de görüldüğü gibi boyutları $(L x W)$ olan dikdörtgen yamanın 1şıma yapmayan kenarlarından birinde, $(l \times{ } \mathrm{x} w)$ boyutlarında bir boşluğun açılmasıyla elde edilen yamanın, toprak düzlem üzerinde bulunan $h$ yüksekliğindeki dielektrik malzemenin üzerine yerleştirilmesi ile oluşturulmuştur [45-48]. Literatürde, $L=50 \mathrm{~mm}$, $W=45 \mathrm{~mm}, l=22 \mathrm{~mm}, w=20 \mathrm{~mm}, h=8 \mathrm{~mm}$ ve $\varepsilon_{r}=1,07$ fiziksel ve elektriksel parametrelerde LKMA'nın rezonans frekansı için benzetim ve ölçüm sonuçları [48] ve DMA'ya göre eşdeğer alan kullanarak iletken boyundaki uzamaya bağlı formüller [46] sunulmuştur.
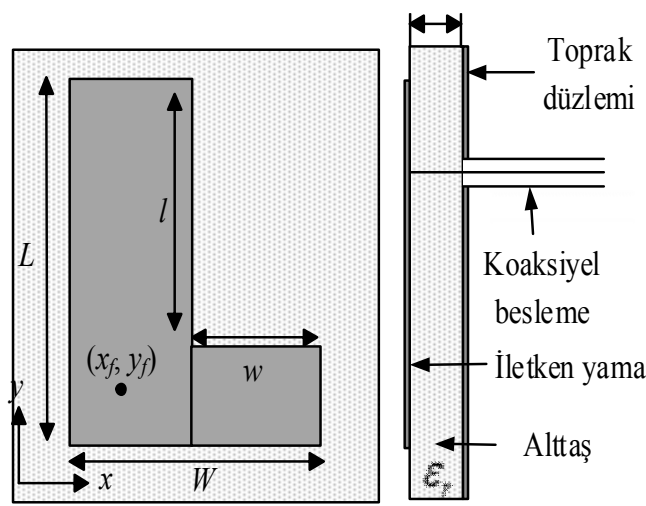

Şekil 7. LKMA geometrisi (Geometry of LCMA) 
Ayrıca, Kayabaşı vd. [40] tarafindan 2014 yılında yapılan çalışma ile LKMA'ların rezonans frekansı hesabı için sırasıyla Tablo 13 ve 14 'de ağ parametreleri verilen YSA ve BMSDUA modelleri önerilmiştir. YSA modeli 3 nöronlu bir gizli katmandan oluşmakta ve giriş ve gizli katmanlar için tanjant sigmoid fonksiyonu, çıkış katmanı için ise purelin fonksiyonu kullanılmıştır. BMSDUA modelinde ise giriş ve çıkış değerleri için üyelik fonksiyonu sayısı 22 olarak seçilmiştir.

Kayabaşı vd., 2014 [40], benzetimleri yapılan farklı fiziksel ve elektriksel parametrelere sahip 192 LKMA'nın 172'sini, YSA ve BMSDUA modellerinin eğitimi için kullanmışlar ve eğitim neticesinde $\mathrm{OYH}$ sırasıyla $\% 0,345$ ve $\% 0,090$ olarak hesaplamışlardır.

Tablo $15^{\prime}$ de elektriksel ve fiziksel parametreleri verilen, eğitim aşamasında ağların görmediği 20 LKMA ise YSA ve BMSDUA'nın başarısını test etmek için kullanılmıştır. Tablo 16'da görüldüğü gibi test neticesinde YSA ve BMSDUA modelleri ile hesaplanan rezonans frekansları ile benzetim sonuçları arasındaki uyum oldukça iyidir ve test için OYH değeri sirasiyla \% 0,533 ve \% 0,461 olarak elde edilmiştir.

LKMA'ların rezonans frekansı hesabı için sunulan bu yaklaşımların doğruluğu ve geçerliliği hem aynı çalışmada ölçümü yapılan LKMA verileri, hem de literatürde [48] benzetimi ve ölçümü yapılan LKMA verileri ile de test edilmiştir. Test neticesinde Tablo 17 'de görüldüğü gibi başarılı sonuçlar alınmıştır.

Tablo 13. LKMA'nın rezonans frekansı hesabında kullanilan YSA parametreleri (The ANN parameters used for computing the resonant frequency of LCMAs)

\begin{tabular}{lc}
\hline Parametreler & Değer \\
\hline Giriş sayısı & 6 \\
Çıkış sayısı & 1 \\
Epok sayısı & 250 \\
Seed değeri & 1446455104 \\
Minimum gradient azalması & $10^{-10}$ \\
Momentum katsayısı $(\mu)$ & 0.0001 \\
$\mu$ değerindeki artış & 4 \\
$\mu$ değerindeki düşüş & 0.1 \\
Maksimum $\mu$ değeri & $10^{10}$ \\
\hline
\end{tabular}

Tablo 14. LKMA'nın rezonans frekansı hesabında kullanilan BMSDUA parametreleri (The ANFIS parameters used for computing the resonant frequency of LCMAs)

\begin{tabular}{lc}
\hline Parametreler & Tip/değer \\
\hline Giriş üyelik fonksiyonu & Gaussian \\
Çıkış üyelik fonksiyonu & Linear \\
Giriş sayısı & 6 \\
Çıkış sayısı & 1 \\
Bulanık kurallar sayısı & 22 \\
Üyelik fonksiyonlar sayısı & 22 \\
Epok & 100 \\
Etki aralığı & 0.5 \\
Sıkıştırma faktörü & 1.25 \\
Kabul oranı parametre & $6 \times 22 \times 2=264$ \\
Geri çevirme oranı & 0.15 \\
Nonlineer & \\
sayısı & $7 \times 22=154$ \\
Lineer parametre sayısı & 317 \\
Düğüm sayısı & 172 \\
Eğitim veri çifti sayısı & \\
\hline
\end{tabular}

Tablo 15. YSA ve BMSDUA modellerinin testi için kullanılan LKMA'ların fiziksel ve elektriksel parametreleri (The physical and electrical parameters of LCMAs used for ANN and ANFIS test process)

\begin{tabular}{|c|c|c|c|c|c|c|c|}
\hline \multirow{2}{*}{$\begin{array}{l}\text { Anten } \\
\text { say1s1 }\end{array}$} & \multicolumn{5}{|c|}{ Yama boyutları (mm) } & \multirow[b]{2}{*}{$\varepsilon_{r}$} & \multirow{2}{*}{$\begin{array}{c}\text { Rezonans frekans1 }(\mathrm{GHz}) \\
\text { Benzetim [40] }\end{array}$} \\
\hline & $L$ & $W$ & $l$ & $w$ & $h$ & & \\
\hline 1 & 30 & 25 & 15 & 8 & 1,57 & 2,33 & 2,969 \\
\hline 2 & 30 & 25 & 25 & 4 & 1,57 & 2,33 & 3,128 \\
\hline 3 & 30 & 25 & 25 & 12 & 1,57 & 2,33 & 2,640 \\
\hline 4 & 30 & 25 & 10 & 16 & 1,57 & 4,5 & 2,200 \\
\hline 5 & 30 & 25 & 10 & 4 & 1,57 & 6,15 & 2,013 \\
\hline 6 & 30 & 25 & 20 & 16 & 1,57 & 6,15 & 1,563 \\
\hline 7 & 30 & 25 & 20 & 16 & 1,57 & 9,8 & 1,244 \\
\hline 8 & 40 & 30 & 25 & 5 & 2,5 & 2,33 & 2,359 \\
\hline 9 & 40 & 30 & 30 & 20 & 2,5 & 2,33 & 1,844 \\
\hline 10 & 40 & 30 & 25 & 5 & 2,5 & 4,5 & 1,713 \\
\hline 11 & 40 & 30 & 35 & 10 & 2,5 & 4,5 & 1,650 \\
\hline 12 & 40 & 30 & 20 & 10 & 2,5 & 6,15 & 1,432 \\
\hline 13 & 40 & 30 & 30 & 20 & 2,5 & 6,15 & 1,183 \\
\hline 14 & 40 & 30 & 20 & 15 & 2,5 & 9,8 & 1,090 \\
\hline 15 & 50 & 35 & 30 & 6 & 3,17 & 2,33 & 1,904 \\
\hline 16 & 50 & 35 & 35 & 18 & 3,17 & 2,33 & 1,635 \\
\hline 17 & 50 & 35 & 35 & 6 & 3,17 & 4,5 & 1,380 \\
\hline 18 & 50 & 35 & 40 & 12 & 3,17 & 4,5 & 1,303 \\
\hline 19 & 50 & 35 & 35 & 12 & 3,17 & 6,15 & 1,114 \\
\hline 20 & 50 & 35 & 45 & 24 & 3,17 & 6,15 & 0,983 \\
\hline
\end{tabular}


Tablo 16. LKMA'lar için benzetim, YSA ve BMSDUA sonuçlarının karşılaştırılması (The comparison of the results of simulation, ANN and ANFIS for LCMAs)

\begin{tabular}{|c|c|c|c|c|c|}
\hline \multirow{2}{*}{$\begin{array}{l}\text { Anten } \\
\text { say1s1 }\end{array}$} & \multicolumn{3}{|c|}{ Rezonans frekansı (GHz) } & \multicolumn{2}{|c|}{ Yüzdelik hata (\%) } \\
\hline & Benzetim [40] & YSA [40] & BMSDUA [40] & $\mathrm{H}_{\mathrm{YSA}}[40]$ & $\mathrm{H}_{\text {BMSDUA }}[40]$ \\
\hline 1 & 2,969 & 2,959 & 2,959 & 0,328 & 0,339 \\
\hline 2 & 3,128 & 3,125 & 3,137 & 0,116 & 0,277 \\
\hline 3 & 2,640 & 2,650 & 2,640 & 0,371 & 0,004 \\
\hline 4 & 2,200 & 2,216 & 2,204 & 0,714 & 0,195 \\
\hline 5 & 2,013 & 2,031 & 2,020 & 0,899 & 0,373 \\
\hline 6 & 1,563 & 1,556 & 1,564 & 0,448 & 0,096 \\
\hline 7 & 1,244 & 1,246 & 1,261 & 0,181 & 1,387 \\
\hline 8 & 2,359 & 2,345 & 2,361 & 0,605 & 0,086 \\
\hline 9 & 1,844 & 1,837 & 1,816 & 0,382 & 1,489 \\
\hline 10 & 1,713 & 1,716 & 1,725 & 0,187 & 0,718 \\
\hline 11 & 1,650 & 1,647 & 1,648 & 0,164 & 0,152 \\
\hline 12 & 1,432 & 1,423 & 1,429 & 0,634 & 0,215 \\
\hline 13 & 1,183 & 1,189 & 1,190 & 0,507 & 0,616 \\
\hline 14 & 1,090 & 1,093 & 1,101 & 0,306 & 0,976 \\
\hline 15 & 1,904 & 1,944 & 1,925 & 2,061 & 1,095 \\
\hline 16 & 1,635 & 1,618 & 1,634 & 1,008 & 0,029 \\
\hline 17 & 1,380 & 1,376 & 1,378 & 0,254 & 0,146 \\
\hline 18 & 1,303 & 1,305 & 1,303 & 0,170 & 0,008 \\
\hline 19 & 1,114 & 1,110 & 1,119 & 0,358 & 0,459 \\
\hline 20 & 0,983 & 0,993 & 0,978 & 0,968 & 0,568 \\
\hline $\mathrm{OYH}$ & & & & 0,533 & 0,461 \\
\hline
\end{tabular}

Tablo 17. LKMA'lar için [40] ve [48] sonuçları ile YSA ve BMSDUA sonuçlarının karşılaştırması (Comparison of the results of [40], [48] simulation and measurement for LCMAs)

\begin{tabular}{cccccccc}
\hline \multirow{2}{*}{ LKMA } & \multicolumn{2}{c}{ Rezonans frekansları $(\mathrm{GHz})$} & & \multicolumn{2}{c}{ Yüzdelik hatalar (\%) } \\
\cline { 2 - 4 } \cline { 6 - 7 } \cline { 6 - 7 } & Ölçüm & YSA & BMSDUA & & YSA & BMSDUA \\
\cline { 1 - 3 }$[46]$ & 3,13 & 3,121 & 3,135 & & 0,542 & 0,096 \\
{$[54]$} & 2,68 & 2,738 & 2,765 & & 0,436 & 0,545 \\
\hline
\end{tabular}

3.5 Dikdörtgen Halka Şekilli Kompakt Mikroşerit Anten (Rectangular Ring Shaped Compact Microstrip Antennas)

Şekil 8'de görüldüğü gibi, DHKMA, $(L \times W)$ boyutlarındaki DMA'nın iletken yamasının merkezinde, simetrik olacak şekilde $(l \times w)$ ölçülerinde dikdörtgen bir boşluk açılması ile elde edilmektedir [11].

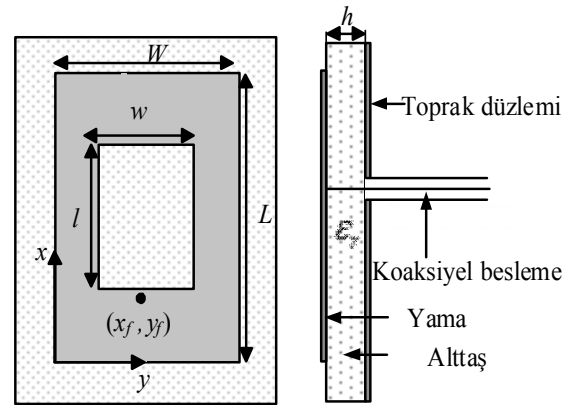

Şekil 8. DHKMA geometrisi (Geometry of RRCMA)

Deshmukh ve Kumar [11], 2007 yılında DHKMA'ların rezonans frekansı hesabı için iletken boyundaki uzamaya bağlı olarak farklı formüller önermişlerdir. $\mathrm{Bu}$ formüller, $L=60 \mathrm{~mm}, W=40 \mathrm{~mm}$, $h=1,59 \mathrm{~mm}$ ve $\varepsilon_{r}=2,33$ fiziksel ve elektriksel parametrelerde, farklı boşluk uzunluklarına sahip 7 benzetim verisi ile test edilmiştir. Akdağlı vd., 2014 [41] tarafindan DHKMA'ların rezonans frekansı hesabı için Tablo 18'de ağ parametreleri verilen BMSDUA modeli önerilmiştir. BMSDUA modelinin eğitimi için IE3D ${ }^{\mathrm{TM}}$ ile benzetimi yapılan 108 DHKMA'nın 96'sı kullanılmış ve OYH \% 0,014 olarak hesaplanmıştır. Benzetimi yapılan 108 DHKMA'nın içinden eğitim aşamasında ağın görmediği ve çözüm uzayını temsil edecek şekilde seçilen 12 anten eğitilen BMSDUA modelinin başarısını test etmek için kullanılmış ve hesaplanan rezonans frekans değerleri Tablo 19'da verilmiştir.

Yapay zeka tekniklerinden BMSDUA modelinin önerildiği bu yaklaşımın geçerliliğini göstermek için literatürde [11] verilen benzetim ve hesaplama sonuçları için de test işlemi uygulanmıştır. Tablo 20'de görüldüğü gibi BMSDUA'nın hesapladığı sonuçlar, Deshmukh ve Kumar, 2007 [11] tarafindan hesaplanan sonuçlardan daha iyidir. Ayrıca BMSDUA modeli [41] aynı çalışmada ölçümü yapılan DHKMA verileri ile de test edilmiş ve Tablo 21 'de görüldüğü başarılı sonuçlar alınmıştır. 
Tablo 18. DHKMA'nın rezonans frekansı hesabında kullanılan BMSDUA parametreleri (The ANFIS parameters used for computing the resonant frequency of RRCMAs)

\begin{tabular}{lc}
\hline Parametreler & Tip/değer \\
\hline Giriş üyelik fonksiyonu & Gaussian \\
Çıış üyelik fonksiyonu & Linear \\
Giriş sayısı & 6 \\
Çıkış sayısı & 1 \\
Bulanık kurallar sayısı & 30 \\
Üyelik fonksiyonlar sayısı & 30 \\
Epok & 100 \\
Etki aralığı & 0,5 \\
Sikıştırma faktörü & 1,25 \\
Kabul oranı & 0,5 \\
Geri çevirme oranı & 0,15 \\
Nonlineer parametre sayısı & $6 \times 30 \times 2=360$ \\
Lineer parametre sayısı & $7 \times 30=210$ \\
Düğüm sayısı & 429 \\
Eğitim veri çifti sayısı & 96 \\
\hline
\end{tabular}

Tablo 19. Test sonucunda BMSDUA ile belirlenen DHKMA rezonans frekanslar1 (The resonant frequencies of RRCMAs determined by ANFIS for test process)

\begin{tabular}{|c|c|c|c|c|c|c|c|c|c|}
\hline \multirow{2}{*}{$\begin{array}{l}\text { Anten } \\
\text { sayis1 }\end{array}$} & \multicolumn{5}{|c|}{ Yama boyutları (mm) } & \multirow[b]{2}{*}{$\varepsilon_{r}$} & \multicolumn{2}{|c|}{ Rezonans frekansları $(\mathrm{GHz})$} & \multirow{2}{*}{$\begin{array}{l}\text { Yüzdelik } \\
\text { hata }(\%) \\
H_{\text {BMSDUA }}\end{array}$} \\
\hline & $L$ & $W$ & $l$ & $\mathrm{~W}$ & $h$ & & Benzetim [41] & BMSDUA [41] & \\
\hline 1 & 30 & 20 & 12 & 8 & 3,175 & 2,2 & 3,153 & 3,155 & 0,063 \\
\hline 2 & 40 & 30 & 10 & 16 & 3,175 & 2,2 & 2,207 & 2,219 & 0,544 \\
\hline 3 & 50 & 40 & 18 & 14 & 3,175 & 2,2 & 1,861 & 1,850 & 0,591 \\
\hline 4 & 60 & 40 & 24 & 18 & 3,175 & 2,2 & 1,506 & 1,505 & 0,066 \\
\hline 5 & 30 & 20 & 8 & 8 & 1,6 & 4,4 & 2,170 & 2,171 & 0,046 \\
\hline 6 & 40 & 30 & 14 & 16 & 1,6 & 4,4 & 1,450 & 1,431 & 1,310 \\
\hline 7 & 50 & 40 & 24 & 18 & 1,6 & 4,4 & 1,199 & 1,218 & 1,585 \\
\hline 8 & 60 & 40 & 24 & 18 & 1,6 & 4,4 & 1,007 & 0,996 & 1,092 \\
\hline 9 & 30 & 20 & 8 & 8 & 0,64 & 10,2 & 1,373 & 1,373 & 0,000 \\
\hline 10 & 40 & 30 & 10 & 16 & 0,64 & 10,2 & 0,910 & 0,923 & 1,429 \\
\hline 11 & 50 & 40 & 18 & 18 & 0,64 & 10,2 & 0,750 & 0,756 & 0,800 \\
\hline 12 & 60 & 40 & 18 & 18 & 0,64 & 10,2 & 0,641 & 0,644 & 0,468 \\
\hline $\mathrm{OYH}$ & & & & & & & & & 0,666 \\
\hline
\end{tabular}

Tablo 20. DHKMA benzetim ve hesaplama sonuçları [11] ile BMSDUA sonuçlarının [41] karşılaştırılması (The comparison of the results of simulation, calculation [11] and ANFIS for RRCMAs)

\begin{tabular}{|c|c|c|c|c|c|c|c|c|c|c|}
\hline \multicolumn{5}{|c|}{$\begin{array}{l}\text { Yama boyutları } \\
(\mathrm{mm})\end{array}$} & \multirow[t]{2}{*}{$\varepsilon_{r}$} & \multicolumn{3}{|c|}{$\begin{array}{c}\text { Rezonans frekansları } \\
\mathrm{GHz})\end{array}$} & \multicolumn{2}{|c|}{$\begin{array}{c}\text { Yüzdelik hata } \\
(\%)\end{array}$} \\
\hline$L$ & $W$ & $l$ & $w$ & $h$ & & Benzetim [11] & Formül [11] & BMSDUA [41] & $\mathrm{H}_{[11]}$ & $\mathrm{H}_{\text {BMSDUA }}$ \\
\hline 60 & 40 & 5 & 5 & 1,59 & 2,33 & 1,593 & 1,643 & 1,538 & 3,139 & 3,471 \\
\hline 60 & 40 & 10 & 10 & 1,59 & 2,33 & 1,534 & 1,525 & 1,517 & 0,587 & 1,121 \\
\hline 60 & 40 & 15 & 15 & 1,59 & 2,33 & 1,428 & 1,425 & 1,424 & 0,210 & 0,308 \\
\hline 60 & 40 & 20 & 20 & 1,59 & 2,33 & 1,315 & 1,338 & 1,305 & 1,749 & 0,798 \\
\hline 60 & 40 & 40 & 20 & 1,59 & 2,33 & 1,345 & 1,274 & 1,370 & 5,279 & 1,874 \\
\hline 60 & 40 & 45 & 25 & 1,59 & 2,33 & 1,260 & 1,293 & 1,287 & 2,619 & 2,175 \\
\hline 60 & 40 & 50 & 30 & 1,59 & 2,33 & 1,210 & 1,164 & 1,230 & 3,802 & 1,620 \\
\hline \multicolumn{5}{|c|}{$\mathrm{OYH}$} & & & & & 2.484 & 1,624 \\
\hline
\end{tabular}

Tablo 21. DHKMA için benzetim, ölçüm ve BMSDUA sonuçlarını karşılaştırılması (The comparison of the results of simulation, measurement and ANFIS for RRCMAs)

\begin{tabular}{|c|c|c|c|c|c|c|c|c|c|}
\hline \multirow{2}{*}{ DHKMA } & \multicolumn{4}{|c|}{ Yama boyutları mm) } & \multirow[b]{2}{*}{$h$} & \multirow[b]{2}{*}{$\varepsilon_{r}$} & \multicolumn{3}{|c|}{ Rezonans frekansı (GHz) } \\
\hline & $\mathrm{L}$ & $\mathrm{W}$ & $l$ & $w$ & & & Benzetim & Ölçüm & BMSDUA \\
\hline [47] & 35 & 25 & 18 & 10,15 & 1,575 & 2,33 & 2,437 & 2,439 & 2,456 \\
\hline
\end{tabular}




\section{SONUÇLAR (CONCLUSIONS)}

Bu çalışmada, CKMA, HKMA, EKMA, LKMA ve DHKMA gibi oyuk yüklü KMA'ların rezonans frekansı hesabı için YSA ve BMSDUA ağlarının kullanıldığı, literatüre sunulmuş çalışmalar ve sonuçları ele alınmıştır. Literatürde sunulan çalışmalarda, YSA ve BMSDUA ağlarının eğitimi ve testi için veriler elektromanyetik benzetim programları kullanılarak elde edilmiştir. Ayrıca YSA ve BMSDUA modellerinin benzetim verilerinin dışında test etmek için daha önce yapılmış çalışmalardaki benzetim ve ölçüm sonuçları da kullanılmış ve literatürde önerilen diğer metotların sonuçları ile de karşılaştırılmıştır. Oyuk yüklü KMA'ların hesaplanan rezonans frekansı ve karşılaştırma sonuçlarına bakıldığında, yapay zeka tekniklerinin, KMA'ların rezonans frekansı hesapları için etkili ve başarılı bir şekilde kullanılabileceği görülmektedir. $\mathrm{Bu}$ çalışmalardan, KMA tasarımcilarının tasarlamak istedikleri antenlerin fiziksel ve elektriksel parametrelerini, YSA ve BMSDUA modellerini kullanarak hazırlanan programlara giriş verisi olarak girerek KMA'ların rezonans frekansını çok fazla zaman gerektirmeden büyük bir doğruluk içinde hesaplayabilecekleri görülmektedir.

\section{KAYNAKLAR (REFERENCES)}

1. Sainati, R. A. CAD of Microstrip Antennas for Wireless Applications, Nowood, MA: Artech House, 1996.

2. Garg, R., Bhartia, P., Bahl, I. ve Ittipiboon, A., Microstrip Antenna Design Handbook, Londra, Artech House, 2001.

3. Kumar, G. ve Ray, K.P. Broadband Microstrip Antennas, Artech House, USA, 2003.

4. Sagiroglu, S. ve Güney, K., "Calculation of Resonant Frequency for an Equilateral Triangular Microstrip Antenna with the Use of Artificial Neural Networks", Microwave and Optical Technology Letters, Cilt 14, No 2, 89-93, 1997.

5. Guney, K. ve Gultekin, S. S., "Artificial Neural Networks for Resonant Frequency Calculation of Rectangular Microstrip Antennas with Thin and Thick Substrates", International Journal of Infrared and Millimeter Waves, Cilt 25, No 9, 1383-1399, 2004.

6. Guney, K. ve Sarikaya, N. "Input Resistance Calculation for Circular Microstrip Antennas Using Adaptive Neuro-Fuzzy Inference System”, International Journal of Infrared and Millimeter Waves, Cilt 25, No 4, 703-716, 2004.

7. Krishna, D. D., Gopikrishna, M., Aanandan, C. K., Mohanan, P. ve Vasudevan, K., "Compact Dual Band Slot Loaded Circular Microstrip Antenna with a Superstrate", Progress In Electromagnetics Research, Cilt 83, 245-255, 2008.
8. Paulson, M., Kundukulam, S.O., Aanandan, C.K. ve Mohanan, P., "Resonance Frequencies of Compact Microstrip Antenna", Electronics Letters, Cilt 37, 1151-1153, 2001.

9. Gao, S.C., Li, L. W., Leong, M. S. ve Yeo, T. S., "Analysis of an H-Shaped Patch Antenna by Using FDTD Method", Progress In Electromagnetics Research, Cilt 34, 165-187, 2001.

10. Sheta, A.F., Mohra, A. ve Mahmoud, S.F., "Multi-Band Operation of a Compact H-Shaped Microstrip Antenna", Microwave and Optical Technology Letters, Cilt 35, 363-367, 2002.

11. Deshmukh, A. A. ve Kumar, G., "Formulation of Resonant Frequency for Compact Rectangular Microstrip Antennas", Microwave and Optical Technology Letters, Cilt 49, No 2, 498-501, 2007.

12. Akdagli, A. Ozdemir, C., Yamacli, S. ve Arcasoy, C. C., "Improved Formulas for the Resonant Frequencies of Dual Frequency Arrow Shaped Compact Microstrip Antenna", Microwave and Optical Technology Letters, Cilt 50, 62-65, 2008.

13. Akdagli, A. ve Toktas, A. "A Novel Expression in Calculating Resonant Frequency of H-Shaped Compact Microstrip Antennas Obtained by Using Artificial Bee Colony Algorithm", Journal of Electromagnetic Waves and Applications, Cilt 24, No 14-15, 2049-4061, 2010.

14. Akdagli, A., Bicer, M.B., ve Ermis, S., "A Novel Expression for Resonant Length Obtained by Using Artificial Bee Colony Algorithm in Calculating Resonant Frequency of C-Shaped Compact Microstrip Antennas", Turkish Journal of Electrical Engineering and Computer Sciences, Cilt 19, 597-606, 2011.

15. Toktas A., Akdagli A., Bicer M.B., ve Kayabasi, A., "Simple Formulas for Calculating Resonant Frequencies of $\mathrm{C}$ and $\mathrm{H}$ Shaped Compact Microstrip Antennas Obtained by Using Artificial Bee Colony Algorithm", Journal of Electromagnetic Waves and Applications, Cilt 25, 1718-1729, 2011.

16. Bhattacharyya, A. K. ve Garg, R., "A Generalized Transmission Line Model for Microstip Patches", IEE Proceedings Microwave Antennas and Propagation, Cilt 132, No 2, 93-98, 1985.

17. Richards, W. F., Lo, Y. T. ve Harrisson, D. D., "An Improved Theory for Microstrip Antennas and Applications", IEEE Transactions on Antennas and Propagation, Cilt 29, 38-46, 1981.

18. Harrington, R. F., Field Computation by Moment Methods, IEEE Press, Piscataway, NJ, 1993.

19. Taflove, A., Computational Electrodynamics: The Finite - Difference Time Domain Method, Boston, Artech House, 1995. 
20. Haykin, S., Neural Networks: A Comprehensive Foundation, Macmillan College Publishing Company, New York, A.B.D., 1994.

21. Turker, N., Gunes, F. ve Yildirim, T., "Artificial Neural Design of Microstrip Antennas", Turkish Journal of Electrical Engineering and Computer, Cilt 14, No 3, 445-453, 2006.

22. Thakare, V. V. ve Singhal, P., "Microstrip Antenna Design Using Artificial Neural Networks", International Journal of RF and Microwave Computer-Aided Engineering, Cilt 20, 76-86, 2010.

23. Tighilt, Y., Bouttout, F. ve Khellaf A., "Modeling and Design of Printed Antennas Using Neural Networks", International Journal of RF and Microwave Computer-Aided Engineering, Cilt 21, 228-233, 2011.

24. Guney, K. ve Sarikaya, N., “Adaptive NeuroFuzzy Inference System for Computing the Resonant Frequency of Circular Microstrip Antennas", The Applied Computational Electromagnetic Society, Cilt 19, No 3, 188197, 2004.

25. Jang, J. S. R., “ANFIS: Adaptive-Network-Based Fuzzy Inference System", IEEE Transactions on Systems, Cilt 23, No 3, 665-685, 1993.

26. Jang, J.-S.R., "Self-Learning Fuzzy Controllers Based on Temporal Backpropagation", IEEE Transactions on Neural Networks, Cilt 3, No 5, 714-723, 1992.

27. Sagiroglu, S., Güney, K. ve Erler, M., "Resonant Frequency Calculation for Circular Microstrip Antennas Using Artificial Neural Networks", International Journal of RF and Microwave Computer-Aided Engineering, Cilt 8, 270-277, 1998.

28. Karaboğa, D., Güney K., Sağıroğlu S. ve Erler M., "Neural Computation of Resonant Frequency of Electrically Thin and Thick Rectangular Microstrip Antennas", IEE Proceedings Microwave Antennas and Propagation, Cilt 146, No 2, 155-159, 1999.

29. Koçer, D., Daire ve Dikdörtgen Geometrik Yapılı Mikroşerit Antenlerin Simülasyonu ve Rezonans Frekanslarının Yapay Sinir Ağları ile Belirlenmesi, Yüksek Lisans Tezi, Selçuk Üniversitesi Fen Bilimleri Enstitüsü, 2009.

30. Merad L., Bendimerad F. T. ve Meriah, S. M., "Design and Resonant Frequency Calculation of Rectangular Microstrip Antennas", International Journal of Numerical Modelling: Electronic Networks, Devices and Fields, Cilt 24, 144-153, 2011.

31. Yildiz, C., Gultekin, S., Guney, K. ve Sagiroglu, S., "Neural Models for the Resonant Frequency of Electrically Thin and Thick Circular Microstrip Antennas and the Characteristic Parameters of Asymmetric Coplanar Waveguides Backed with a Conductor", International
Journal of Electronics and Communications, Cilt 56, No 6, 396-406, 2002.

32. Brinhole, E. R. Destro, J. F. Z., de Freitas, A. A. C. ve de Alcantara, N. P. Jr., "Determination of Resonant Frequencies of Triangular and Rectangular Microstrip Antennas, Using Artificial Neural Networks", Progress In Electromagnetics Research Symposium, Hangzhou, China, 22-26, 579-582, 2005.

33. Gupta, P., Gupta, R., Sharma, R. ve Yadav, B., "Calculation of Resonating Frequency of an Equilateral Triangular Microstrip Antenna Using Artificial Neural Network", Advanced Computational Techniques in Electromagnetics, Article ID acte-00126, 8 Pages doi: 10.5899/2013/acte-00126, 2013.

34. Ouchar, A., Aksas, R. and Baudrand, H., "Artificial Neural Network for Computing the Resonant Frequency of Circular Patch Antennas," Microwave and Optical Technology Letters, Cilt 47, No 6, 564-566, 2005.

35. Sarıkaya Baştürk N., Bulanik Mantik Sistemleri ile Çeşitli Tipteki Mikroşerit Antenlerin Karakteristik Parametrelerinin Hesaplanmasi, Doktora Tezi, Erciyes Üniversitesi, Fen Bilimleri Enstitüsü, 2009.

36. Angiulli, G. ve Versaci, M., "Resonant Frequency Evaluation of Microstrip Antennas Using a Neural-Fuzzy Approach", IEEE Transactions on Magnetics, Cilt 39, No 3, 1333-1336, 2003.

37. Akdagli A., Kayabasi A. ve Develi İ., "Computing Resonant Frequency of C-Shaped Compact Microstrip Antennas by Using ANFIS", International Journal of Electronics, Cilt 102, No 3, 407-417, 2014.

38. Kayabasi A., Bicer M. B., Akdagli A. ve Toktas A., "Computing Resonant Frequency of HShaped Compact Microstrip Antennas Operating at UHF Band by Using Artificial Neural Networks", Journal of the Faculty of Engineering and Architecture of Gazi University, Cilt 26, No 4, 833-840, 2011.

39. Akdagli A., Toktas A., Kayabasi A. ve Develi İ., " An Application of Artificial Neural Network to Compute the Resonant Frequency of E-Shaped Compact Microstrip Antennas", Journal of Electrical Engineering- Elektrotechnicky Casopis, Cilt 64, No 5, 317-322, 2013.

40. Kayabasi A., Toktas A., Akdagli A., Bicer M. B. ve Ustun D., "Applications of ANN and ANFIS to Predict the Resonant Frequency of L-shaped Compact Microstrip Antennas", The Applied Computational Electromagnetic Society, Cilt 29, No 6, 460-469, 2014.

41. Akdagli A., Toktas A., Bicer M. B., Kayabasi A., Ustun D., ve Kurt K., "ANFIS Model for Determining Resonant Frequency of Rectangular Ring Compact Microstrip Antennas", International Journal of Applied 
Electromagnetics Mechanics, in the status of pre-press, Cilt 46, 483-490, 2014.

42. Ooi B. L ve Shen Q., "A Novel E-Shaped Broadband Microstrip Patch Antenna", Microwave and Optical Technology Letters, Cilt 27, No 5, 348-352, 2000.

43. Yang, F., Zhang, X. X., Ye, X. N. ve RahmatSamii, Y., "Wide-Band E-Shaped Patch Antennas for Wireless Communications", IEEE Transactions on Antennas and Propagation, Cilt 49, 1094-1100, 2001.

44. Neog, D. K., Pattnaik, S. S., Panda, D. C., Devi, S., Dutta, M. ve Bajpai, O. P., "New Expression for the Resonance Frequency of an E-Shaped Microstrip Patch Antenna", Microwave and Optical Technology Letters, Cilt 48, 1561-1563, 2006.
45. Neog, D. K. ve Devi, R., "Determination of Resonant Frequency of Slot-Loaded Rectangular Microstrip Patch Antennas", Microwave and Optical Technology Letters, Cilt 52, No 2, 446448, 2010.

46. Gunel, T., "Modified Resonant Frequency Calculation for E-Shaped and H-Shaped Microstrip Patch Antennas", Microwave and Optical Technology Letters, Cilt 53, 2348-2351, 2011.

47. Toktas A. ve Akdagli A., "Computation of Resonant Frequency of E-Shaped Compact Microstrip Antennas", Journal of the Faculty of Engineering and Architecture of Gazi University, Cilt 27, 847-854, 2012.

48. Chen, Z.N., "Radiation Pattern of A Probe Fed LShaped Plate Antenna", Microwave and Optical Technology Letters, Cilt 27, 410-413, 2000. 
